http://dx.doi.org/10.17776/csj.04266

\title{
Some Properties of Matrix Algebra of Semi-quaternions
}

\author{
Mehdi JAFARI and Habib MOLAEI \\ 1 Department of Mathematics, University College of Science and Technology Elm O Fan, Urmia, Iran \\ 2 University Technology Gazi Tabatabaei, P.O Box 57169-33959, Urmia, Iran
}

\begin{abstract}
By representing semi-quaternions as four-dimensional vectors and the multiplication of quaternions as matrix-by-vector product, we investigate properties of matrix associated with a semi-quaternion and examine De-Moivre's formula for this matrix, from which the $n$-th power of such a matrix can be determined.
\end{abstract}

Mathematics Subject Classification(2010): 11R52

Key words: De Moivre's formula, Hamilton operator, semi-quaternion

\section{IntroduCtion}

The quaternion number system was discovered by Hamilton [6], who was looking for an extension of the complex number system to use in various areas of mathematics. Recently, quaternions have developed a wide-spread use in computer graphics and robotics research because they are the simplest algebraic tool for describing rotations in three and four dimensions. Certainly, the numbers have fallen short of the early expectations of the quaternionists. The Euler's and De-Moivre's formulas for the complex numbers are generalized for quaternions [3]. Using De Moivre's formula to find roots of a quaternion is more useful way. The Euler's and De Moivre's formulas are also investigated for the case of dual, split and generalized quaternions in $[9,11,12,14]$.

Some algebraic properties of Hamilton operators are considered in [2] where real quaternions have been expressed in terms of $4 \times 4$ matrices by means of these operators. These matrices have applications in many fields, such as mechanics, quantum physics and computer-aided geometric design [1]. Eigenvalues, eigenvectors and the others algebraic properties of these matrices are studied by several authors [5, 17]. In addition to, Yayl has considered homothetic motions with aid of the Hamilton operators in four-dimensional Euclidean space $E^{4}[16]$. Recently, we have derived the De-Moivre's and Euler's formulas for matrices associated with real, dual and generalized quaternions and every power of these matrices are immediately obtained $[7,8,10]$.

Some algebraic properties of semi-quaternions is investigated by Mortezaasl and Jafari [13]. Euler and De-Moivre's formulas for semi-quaternions are expressed in [13] and the roots of a unit semi-quaternion are given. Here, after a review of some properties of semi-quaternions, De Moivre's and Euler's formulas for the matrices associated with these quaternions are studied. Furthermore, the $n$-th roots of these matrices are derived. Finally, we give some examples for more clarification.

\section{Preliminaries}

We start with some information on the algebra of quaternions and semi-quaternions. For detailed information about these concepts, we refer the reader to [13,15].

\footnotetext{
* Corresponding author. Email address: mjafari@science.ankara.edu.tr 


\section{Some Properties of Matrix Algebra of Semi-quaternions}

Definition 2.1. A real quaternion is defined as

$$
q=a_{\circ}+a_{1} i+a_{2} j+a_{3} k
$$

where $a_{\circ}, a_{1}, a_{2}$ and $a_{3}$ are real numbers and $1, i, j, k$ of $q$ may be interpreted as the four basic vectors of Cartesian set of coordinates; and they satisfy the noncommutative multiplication rules

$$
\begin{aligned}
& i^{2}=j^{2}=k^{2}=i j k=-1 \\
& i j=k=-j i, \quad j k=i=-k j
\end{aligned}
$$

and

$$
k i=j=-i k \text {. }
$$

A quaternion may be defined as a pair $\left(S_{q}, V_{q}\right)$, where $S_{q}=a_{\circ} \in \mathbb{R}$ is scalar part and $V_{q}=a_{1} i+a_{2} j+a_{3} k \in \mathbb{R}^{3}$ is the vector part of $q$. The quaternion product of two quaternions $p$ and $q$ is defined as

$$
p q=S_{p} S_{q}-\left\langle V_{p}, V_{q}\right\rangle+S_{p} V_{q}+S_{q} V_{p}+V_{p} \wedge V_{q}
$$

where" $\langle$,$\rangle "and " \wedge$ " are the inner and vector products in $\mathbb{R}^{3}$, respectively. The norm of a quaternion is given by the sum of the squares of its components: $N_{q}=$ $a_{\circ}^{2}+a_{1}^{2}+a_{2}^{2}+a_{3}^{2}, N_{q} \in \mathbb{R}$. It can also be obtained by multiplying the quaternion by its conjugate, in either order since a quaternion and its conjugate commute: $N_{q}=\bar{q} q=q \bar{q}$. Every non-zero quaternion has a multiplicative inverse given by its conjugate divided by its norm: $q^{-1}=\frac{\bar{q}}{N_{q}}$. The quaternion algebra $H$ is a normed division algebra, meaning that for any two quaternions $p$ and $q, N_{p q}=N_{p} N_{q}$, and the norm of every non-zero quaternion is non-zero (and positive) and therefore the multiplicative inverse exists for any non-zero quaternion. Of course, as is well known, multiplication of quaternions is not commutative, so that in general for any two quaternions $p$ and $q, p q \neq q p$. This can have subtle ramifications, for example: $(p q)^{2}=p q p q \neq p^{2} q^{2}$.

\section{SEMI-QUATERNIONS Algebra}

Definition 3.1. A semi-quaternion $q$ has a expression of form

$$
q=a_{\circ}+a_{1} i+a_{2} j+a_{3} k
$$

where $a_{\circ}, a_{1}, a_{2}$ and $a_{3}$ are real numbers and $i, j, k$ are quaternionic units which satisfy the equalities

$$
\begin{aligned}
& i^{2}=-1, \quad j^{2}=k^{2}=0 \\
& i j=k=-j i, \quad j k=0=k j
\end{aligned}
$$

and

$$
k i=j=-i k
$$

The set of all semi-quaternions are denoted by $H_{s}$. A semi-quaternion $q$ is a sum of a scalar and a vector, called scalar part, $S_{q}=a_{\circ}$, and vector part $V_{q}=$ $a_{1} i++a_{2} j+a_{3} k$. Also, a semi-quaternion can be represented in the following way; $q=z_{1}+i z_{2}$, where $z_{1}=a_{\circ}+a_{2} j, z_{2}=a_{1}+a_{3} j$. 


\section{Jafari and Molaei}

The addition rule for semi-quaternions is component-wise addition:

$$
\begin{aligned}
q+p & =\left(a_{\circ}+a_{1} i+a_{2} j+a_{3} k\right)+\left(b_{\circ}+b_{1} i+b_{2} j+b_{3} k\right) \\
& =\left(a_{\circ}+b_{\circ}\right)+\left(a_{1}+b_{1}\right) i+\left(a_{2}+b_{2}\right) j+\left(a_{3}+b_{3}\right) k .
\end{aligned}
$$

This rule preserves the associativity and commutativity properties of addition. The product of scalar and a semi-quaternion is defined in a straightforward manner. If $c$ is a scaler and $q \in H_{s}$,

$$
c q=c S_{q}+c V_{q}=\left(c a_{\circ}\right) 1+\left(c a_{1}\right) i+\left(c a_{2}\right) j+\left(c a_{3}\right) k .
$$

The multiplication rule for semi-quaternions is defined as

$$
q p=S_{q} S_{p}-g\left(V_{q}, V_{p}\right)+S_{q} V_{p}+S_{p} V_{q}+V_{p} \times V_{q}
$$

where

$$
g\left(V_{q}, V_{p}\right)=a_{1} b_{1}, V_{p} \times V_{q}=0 i+\left(a_{3} b_{1}-a_{1} b_{3}\right) j+\left(a_{1} b_{2}-a_{2} b_{1}\right) k .
$$

Also, this can be written as

$$
p q=\left[\begin{array}{cccc}
a_{\circ} & -a_{1} & 0 & 0 \\
a_{1} & a_{\circ} & 0 & 0 \\
a_{2} & a_{3} & a_{\circ} & -a_{1} \\
a_{3} & -a_{2} & a_{1} & a_{\circ}
\end{array}\right]\left[\begin{array}{l}
b_{\circ} \\
b_{1} \\
b_{2} \\
b_{3}
\end{array}\right] .
$$

Obviously, the quaternion multiplication is associative and distributive with respect to addition and subtraction, but the commutativity law does not hold in general.

Corollary 3.1. $H_{s}$ with addition and multiplication has all the properties of a number field expect commutativity of the multiplication. It is therefore called the skew field of quaternions.

\section{De Moivre's Formula for Semi-QAuternions}

In this section, we express the semi-quaternion $q$ in polar form and look for also De-Moivre's formula for any semi-quaternions (see [13]).

Definition 4.1. Every nonzero semi-quaternion can be written in the polar form

$$
\begin{aligned}
q & =a_{\circ}+a_{1} i+a_{2} j+a_{3} k \\
q & =r(\cos \varphi+\vec{w} \sin \varphi), \quad 0 \leq \varphi \leq 2 \pi
\end{aligned}
$$

where $r=\sqrt{N_{q}}$ and

$$
\cos \varphi=\frac{a_{\circ}}{r}, \quad \sin \varphi=\frac{\sqrt{a_{1}^{2}}}{r}=\frac{\left|a_{1}\right|}{\sqrt{a_{\circ}^{2}+a_{1}^{2}}} .
$$

The unit vector $\vec{w}$ is given by

$$
\vec{w}=\frac{1}{\sqrt{a_{1}^{2}}}\left(a_{1} i+a_{2} j+a_{3} k\right), a_{1} \neq 0 .
$$




\section{Some Properties of Matrix Algebra of Semi-quaternions}

Since $\vec{w}^{2}=-1$, We have a natural generalization of Euler's formula for unit semi-quaternions

$$
\begin{aligned}
e^{\vec{w} \varphi} & =1+\vec{w} \varphi+\frac{(\vec{w} \varphi)^{2}}{2 !}+\frac{(\vec{w} \varphi)^{3}}{3 !}+\ldots \\
& =1-\frac{\varphi^{2}}{2 !}+\frac{\varphi^{4}}{4 !}-\ldots+\vec{w}\left(\varphi-\frac{\varphi^{3}}{3 !}+\frac{\varphi^{5}}{5 !}-\ldots\right) \\
& =\cos \varphi+\vec{w} \sin \varphi
\end{aligned}
$$

for any real number $\varphi$.

Lemma 4.1. Let $\vec{w}$ be a unit vector, then we have

$$
(\cos \varphi+\vec{w} \sin \varphi)(\cos \psi+\vec{w} \sin \psi)=\cos (\varphi+\psi)+\vec{w} \sin (\varphi+\psi)
$$

Theorem 4.2. (De-Moivre's formula) Let $q=e^{\vec{w} \varphi}=\cos \varphi+\vec{w} \sin \varphi$ be a unit semi-quaternion. Then for every integer $n$;

$$
q^{n}=\cos n \varphi+\vec{w} \sin n \varphi .
$$

Proof. We use induction on positive integers $n$. Assume that $q^{n}=\cos n \varphi+\vec{w} \sin n \varphi$ holds. Then

$$
\begin{aligned}
q^{n+1} & =(\cos \varphi+\vec{w} \sin \varphi)^{n}(\cos \varphi+\vec{w} \sin \varphi) \\
& =(\cos n \varphi+\vec{w} \sin n \varphi)(\cos \varphi+\vec{w} \sin \varphi) \\
& =\cos (n \varphi+\varphi)+\vec{w} \sin (n \varphi+\varphi) \\
& =\cos (n+1) \varphi+\vec{w} \sin (n+1) \varphi
\end{aligned}
$$

The formula holds for all integer $n$, since

$$
\begin{aligned}
q^{-1} & =\cos \varphi-\vec{w} \sin \varphi \\
q^{-n} & =\cos (-n \varphi)+\vec{w} \sin (-n \varphi) \\
& =\cos n \varphi-\vec{w} \sin n \varphi
\end{aligned}
$$

Example 4.1. Let $q=-1+i-j+2 k=\sqrt{2}\left(\cos \frac{3 \pi}{4}+\vec{w} \sin \frac{3 \pi}{4}\right)$ be a semiquaternion. Every powers of this quaternion are found with the aid of the Theorem 4.1. For example, 10-th power is

$$
\begin{aligned}
q^{10} & =2^{5}\left[\cos 10\left(\frac{3 \pi}{4}\right)+\vec{w} \sin 10\left(\frac{3 \pi}{4}\right)\right] \\
& =2^{5}(0-\vec{w}) .
\end{aligned}
$$

Corollary 4.3. There are uncountably many unit semi-quaternions satisfying $q^{n}=$ 1 for every integer $n \geq 3$.

Proof. For every unit vector $\vec{w}$, the quaternion $q=\cos \frac{2 \pi}{n}+\vec{w} \sin \frac{2 \pi}{n}$ is of order $n$. For $n=1$ or $n=2$, the quaternion $q$ is independent of $\vec{w}$. 


\section{Jafari and Molaei}

Example 4.2. $q=\frac{1}{\sqrt{2}}+\left(\frac{1}{\sqrt{2}}, 1,-1\right)=\cos \frac{\pi}{4}+\vec{w} \sin \frac{\pi}{4}$ is of order 8 and $q=$ $\frac{1}{2}+\left(\frac{\sqrt{3}}{2}, 1,1\right)=\cos \frac{\pi}{3}+\vec{w} \sin \frac{\pi}{3}$ is of order 6 .

Theorem 4.4. Let $q=\cos \varphi+\vec{w} \sin \varphi$ be a unit semi-quaternion. The equation $x^{n}=q$ has $n$ roots

$$
x_{k}=\cos \frac{\varphi+2 k \pi}{n}+\vec{w} \sin \frac{\varphi+2 k \pi}{n}, \quad k=0,1, \ldots, n-1 .
$$

Proof. If $x^{n}=q, q$ will have the same unit vector as $x$. So, we assume that $x=\cos \varkappa+\vec{w} \sin \varkappa$ is a root of the equation $x^{n}=q$. From the Theorem 4.1, we have

$$
x^{n}=\cos n \varkappa+\vec{w} \sin n \varkappa,
$$

Thus, we find

$$
\cos n \varkappa=\cos \varphi \quad \& \quad \sin n \varkappa=\sin \varphi .
$$

So, the $n$-th roots of $q$ are $x=\cos \frac{\varphi+2 k \pi}{n}+\vec{w} \sin \frac{\varphi+2 k \pi}{n}$ for $k=0,1, \ldots, n-1$.

Example 4.3. Let $q=1+i-2 j+2 k=\sqrt{2}(\cos \varphi+\vec{w} \sin \varphi)$ be a semi-quaternion. The equation $x^{3}=q$ has 3 roots and these are

$$
x_{k}=\sqrt[6]{2}\left(\cos \frac{\varphi+2 k \pi}{3}+\vec{w} \sin \frac{\varphi+2 k \pi}{3}\right), \quad k=0,1,2 .
$$

So, $x_{0}=\sqrt[6]{2}\left(\cos \frac{\pi}{12}+\vec{w} \sin \frac{\pi}{12}\right), x_{1}=\sqrt[6]{2}\left(\cos \frac{3 \pi}{4}+\vec{w} \sin \frac{3 \pi}{4}\right), x_{2}=\sqrt[6]{2}\left(\cos \frac{17 \pi}{12}+\right.$ $\vec{w} \sin \frac{17 \pi}{12}$ ) are the cube roots of $q$.

Theorem 4.5. Let $q$ be a unit semi-quaternion with the polar form $q=\cos \varphi+$ $\vec{w} \sin \varphi$. If $m=\frac{2 \pi}{\varphi} \in \mathbb{Z}^{+}-\{1\}$, then $n \equiv p(\bmod m)$ is possible if and only if $q^{n}=q^{p}$.

Proof. Let $n \equiv p(\bmod m)$. Then we have $n=a m+p$, where $a \in \mathbb{Z}$.

$$
\begin{aligned}
q^{n} & =\cos n \varphi+\vec{w} \sin n \varphi \\
& =\cos (a m+p) \varphi+\vec{w} \sin (a m+p) \varphi \\
& =\cos \left(a \frac{2 \pi}{\varphi}+p\right) \varphi+\vec{w} \sin \left(a \frac{2 \pi}{\varphi}+p\right) \varphi \\
& =\cos (p \varphi+2 \pi a)+\vec{w} \sin (p \varphi+2 \pi a) \\
& =\cos p \varphi+\vec{w} \sin p \varphi \\
& =q^{p}
\end{aligned}
$$

Now suppose $q^{n}=\cos n \varphi+\vec{w} \sin n \varphi$ and $q^{p}=\cos p \varphi+\vec{w} \sin p \varphi$. Since $q^{n}=q^{p}$, we have $\cos n \varphi=\cos p \varphi$ and $\sin n \varphi=\sin p \varphi$, which means that $n \varphi=p \varphi+2 \pi a$, $a \in \mathbb{Z}$. Thus $n=a \frac{2 \pi}{\varphi}+p, n \equiv p(\bmod m)$. 


\section{Some Properties of Matrix Algebra of Semi-quaternions}

Example 4.4. Let $q=\frac{1}{2}+\left(\frac{\sqrt{3}}{2},-1,2\right)$ be a unit semi-quaternion. From the Theorem $4.3, m=\frac{2 \pi}{\pi / 3}=6$, so we have

$$
\begin{aligned}
q= & q^{7}=q^{13}=\ldots \\
q^{2}= & q^{8}=q^{14}=\ldots \\
q^{3}= & q^{9}=q^{15}=\ldots=-1 \\
& \\
q^{6}= & q^{12}=q^{18}=\ldots=1 .
\end{aligned}
$$

\section{De Moivre's Formula for Matrices of semi-Qauternions}

In this section, we introduce the $\mathbb{R}$-linear transformations representing left multiplication in $H_{s}$ and look for also the De-Moiver's formula for corresponding matrix representation. Let $q$ be a semi-quaternion, then $\varphi_{l}: H_{s} \rightarrow H_{s}$ defined as follows:

$$
\varphi_{l}(x)=q x, \quad x \in H_{s} .
$$

The Hamilton's operator $\varphi_{l}$, could be represented as the matrices;

$$
A_{\varphi_{l}}=\left[\begin{array}{cccc}
a_{\circ} & -a_{1} & 0 & 0 \\
a_{1} & a_{\circ} & 0 & 0 \\
a_{2} & a_{3} & a_{\circ} & -a_{1} \\
a_{3} & -a_{2} & a_{1} & a_{\circ}
\end{array}\right]
$$

We can express the matrix $A_{\varphi_{l}}$ in polar form. Let $q$ be a unit semi-quaternion. Since

$$
\begin{aligned}
q & =a_{\circ}+a_{1} i+a_{2} j+a_{3} k \\
& =\cos \varphi+\vec{w} \sin \varphi \\
& =\cos \varphi+\left(w_{1}, w_{2}, w_{3}\right) \sin \varphi \\
& =\cos \varphi+\left(w_{1} \sin \varphi, w_{2} \sin \varphi, w_{3} \sin \varphi\right)
\end{aligned}
$$

we have

$$
\left[\begin{array}{cccc}
a_{\circ} & -a_{1} & 0 & 0 \\
a_{1} & a_{\circ} & 0 & 0 \\
a_{2} & a_{3} & a_{\circ} & -a_{1} \\
a_{3} & -a_{2} & a_{1} & a_{\circ}
\end{array}\right]=\left[\begin{array}{cccc}
\cos \varphi & -w_{1} \sin \varphi & 0 & 0 \\
w_{1} \sin \varphi & \cos \varphi & 0 & 0 \\
w_{2} \sin \varphi & w_{3} \sin \varphi & \cos \varphi & w_{1} \sin \varphi \\
w_{3} \sin \varphi & -w_{2} \sin \varphi & w_{1} \sin \varphi & \cos \varphi
\end{array}\right]
$$

Theorem 5.1. (De-Moivre's formula for matrices) Let $q=e^{\vec{w} \varphi}=\cos \varphi+\vec{w} \sin \varphi$ be a unit semi-quaternion. For an integer $n$

$$
A=\left[\begin{array}{cccc}
\cos \varphi & -w_{1} \sin \varphi & 0 & 0 \\
w_{1} \sin \varphi & \cos \varphi & 0 & 0 \\
w_{2} \sin \varphi & w_{3} \sin \varphi & \cos \varphi & w_{1} \sin \varphi \\
w_{3} \sin \varphi & -w_{2} \sin \varphi & w_{1} \sin \varphi & \cos \varphi
\end{array}\right]
$$

the $n$-th power of the matrix $A$ reads

$$
A^{n}=\left[\begin{array}{cccc}
\cos n \varphi & -w_{1} \sin n \varphi & 0 & 0 \\
w_{1} \sin n \varphi & \cos n \varphi & 0 & 0 \\
w_{2} \sin n \varphi & w_{3} \sin n \varphi & \cos n \varphi & w_{1} \sin n \varphi \\
w_{3} \sin n \varphi & -w_{2} \sin n \varphi & w_{1} \sin n \varphi & \cos n \varphi
\end{array}\right]
$$




\section{Jafari and Molaei}

Proof. The proof follows immediately from the induction.

Example 5.1. Let $q=-1+i-j+2 k=\sqrt{2}\left(\cos \frac{3 \pi}{4}+\vec{w} \sin \frac{3 \pi}{4}\right)$ be a semi-quaternion. The matrix corresponding to this quaternion is

$$
A=\left[\begin{array}{cccc}
-1 & -1 & 0 & 0 \\
-1 & -1 & 0 & 0 \\
-1 & 2 & -1 & -1 \\
2 & 1 & 1 & -1
\end{array}\right]
$$

every powers of this matix are found to be with the aid of Theorem 5.1, for example, 10-th power is

$$
A^{10}=2^{5}\left[\begin{array}{cccc}
0 & -1 & 0 & 0 \\
-1 & 0 & 0 & 0 \\
-1 & 2 & 0 & -1 \\
2 & 1 & 1 & 0
\end{array}\right]
$$

and 164-th power is

$$
A^{164}=2^{82}\left[\begin{array}{cccc}
-1 & 0 & 0 & 0 \\
0 & -1 & 0 & 0 \\
0 & 0 & -1 & 0 \\
0 & 0 & 0 & -1
\end{array}\right]=-2^{82} I_{4}
$$

\section{Euler's Formula for matrices aCCOSIATEd SEMI-QUATERnions}

Let $A$ be a matrix. We choose

$$
A=\left[\begin{array}{cccc}
0 & -u_{1} & 0 & 0 \\
u_{1} & 0 & 0 & 0 \\
u_{2} & u_{3} & 0 & -u_{1} \\
u_{3} & -u_{2} & u_{1} & 0
\end{array}\right]
$$

then one immediately finds $A^{2}=-I$. We have a netural generalization of Euler's formula for matrix $A$;

$$
\begin{aligned}
e^{A \theta} & =I_{4}+A \varphi+\frac{(A \varphi)^{2}}{2 !}+\frac{(A \varphi)^{3}}{3 !}+\frac{(A \varphi)^{4}}{4 !}+\ldots \\
& =I_{4}+A \varphi \\
& =\cos \varphi+A \sin \varphi \\
& =\left[\begin{array}{cccc}
\cos \varphi & -u_{1} \sin \varphi & 0 & 0 \\
u_{1} \sin \varphi & \cos \varphi & 0 & 0 \\
u_{2} \sin \varphi & u_{3} \sin \varphi & \cos \varphi & u_{1} \sin \varphi \\
u_{3} \sin \varphi & -u_{2} \sin \varphi & u_{1} \sin \varphi & \cos \varphi
\end{array}\right] .
\end{aligned}
$$




\section{Some Properties of Matrix Algebra of Semi-quaternions}

\section{7. $n$ - th Root of Matrices of Semi-Quaternions}

Let $q=\cos \varphi+\vec{w} \sin \varphi$ be a unit semi-quaternions. The matrix accossiated with this quaternion $q$ is of the form (1.1). In a more general case, we substitute the matrix (1.1) by

$$
A=\left[\begin{array}{cccc}
\cos (\theta+2 k \pi) & -u_{1} \sin (\theta+2 k \pi) & 0 & 0 \\
u_{1} \sin (\theta+2 k \pi) & \cos (\theta+2 k \pi) & 0 & 0 \\
u_{2} \sin (\theta+2 k \pi) & u_{3} \sin (\theta+2 k \pi) & \cos (\theta+2 k \pi) & u_{1} \sin (\theta+2 k \pi) \\
u_{3} \sin (\theta+2 k \pi) & -u_{2} \sin (\theta+2 k \pi) & u_{1} \sin (\theta+2 k \pi) & \cos (\theta+2 k \pi)
\end{array}\right],
$$

where $k \in \mathbb{Z}$. The equation $x^{n}=A$ has $n$ roots and they are as follows

$$
A_{k}^{\frac{1}{n}}=\left[\begin{array}{cccc}
\cos \left(\frac{\theta+2 k \pi}{n}\right) & -u_{1} \sin \left(\frac{\theta+2 k \pi}{n}\right) & 0 & 0 \\
u_{1} \sin \left(\frac{\theta+2 k \pi}{n}\right) & \cos \left(\frac{\theta+2 k \pi}{n}\right) & 0 & 0 \\
u_{2} \sin \left(\frac{\theta+2 k \pi}{n}\right) & u_{3} \sin \left(\frac{\theta+2 k \pi}{n}\right) & \cos \left(\frac{\theta+2 k \pi}{n}\right) & u_{1} \sin \left(\frac{\theta+2 k \pi}{n}\right) \\
u_{3} \sin \left(\frac{\theta+2 k \pi}{n}\right) & -u_{2} \sin \left(\frac{\theta+2 k \pi}{n}\right) & u_{1} \sin \left(\frac{\theta+2 k \pi}{n}\right) & \cos \left(\frac{\theta+2 k \pi}{n}\right)
\end{array}\right] .
$$

For $k=0$, the first root is

$$
A_{\circ}^{\frac{1}{n}}=\left[\begin{array}{cccc}
\cos \left(\frac{\theta}{n}\right) & -u_{1} \sin \left(\frac{\theta}{n}\right) & 0 & 0 \\
u_{1} \sin \left(\frac{\theta}{n}\right) & \cos \left(\frac{\theta}{n}\right) & 0 & 0 \\
u_{2} \sin \left(\frac{\theta}{n}\right) & u_{3} \sin \left(\frac{\theta}{n}\right) & \cos \left(\frac{\theta}{n}\right) & u_{1} \sin \left(\frac{\theta}{n}\right) \\
u_{3} \sin \left(\frac{\theta}{n}\right) & -u_{2} \sin \left(\frac{\theta}{n}\right) & u_{1} \sin \left(\frac{\theta}{n}\right) & \cos \left(\frac{\theta}{n}\right)
\end{array}\right]
$$

and for $k=1$, the second root is

$$
A_{1}^{\frac{1}{n}}=\left[\begin{array}{cccc}
\cos \left(\frac{\theta+2 \pi}{n}\right) & -u_{1} \sin \left(\frac{\theta+2 \pi}{n}\right) & 0 & 0 \\
u_{1} \sin \left(\frac{\theta+2 \pi}{n}\right) & \cos \left(\frac{\theta+2 \pi}{n}\right) & 0 & 0 \\
u_{2} \sin \left(\frac{\theta+2 \pi}{n}\right) & u_{3} \sin \left(\frac{\theta+2 \pi}{n}\right) & \cos \left(\frac{\theta+2 \pi}{n}\right) & u_{1} \sin \left(\frac{\theta+2 \pi}{n}\right) \\
u_{3} \sin \left(\frac{\theta+2 \pi}{n}\right) & -u_{2} \sin \left(\frac{\theta+2 \pi}{n}\right) & u_{1} \sin \left(\frac{\theta+2 \pi}{n}\right) & \cos \left(\frac{\theta+2 \pi}{n}\right)
\end{array}\right],
$$

similarly, for $k=n-1$, we obtain the $n$-th root.

Example 7.1. Let $q=-\frac{1}{2}+\frac{\sqrt{3}}{2} i+j+k$ be a unit semi-quaternion. The matrix corresponding to this quaternion is

$$
A=\left[\begin{array}{cccc}
-\frac{1}{2} & -\frac{\sqrt{3}}{2} & 0 & 0 \\
\frac{\sqrt{3}}{2} & -\frac{1}{2} & 0 & 0 \\
1 & 1 & -\frac{1}{2} & -\frac{\sqrt{3}}{2} \\
1 & -1 & \frac{\sqrt{3}}{2} & -\frac{1}{2}
\end{array}\right]
$$

The square roots of the matrix $A$ can be calculated as follows:

$$
A_{k}^{\frac{1}{2}}=\left[\begin{array}{cccc}
\cos \left(\frac{2 k \pi+\frac{2 \pi}{3}}{2}\right) & -u_{1} \sin \left(\frac{2 k \pi+\frac{2 \pi}{3}}{2}\right) & 0 & 0 \\
u_{1} \sin \left(\frac{2 k \pi+\frac{2 \pi}{3}}{2}\right) & \cos \left(\frac{2 k \pi+\frac{2 \pi}{3}}{2}\right) & 0 & 0 \\
u_{2} \sin \left(\frac{2 k \pi+\frac{2 \pi}{3}}{2}\right) & u_{3} \sin \left(\frac{2 k \pi+\frac{2 \pi}{3}}{2}\right) & \cos \left(\frac{2 k \pi+\frac{2 \pi}{3}}{2}\right) & -u_{1} \sin \left(\frac{2 k \pi+\frac{2 \pi}{3}}{2}\right) \\
u_{3} \sin \left(\frac{2 k \pi+\frac{2 \pi}{3}}{2}\right) & -u_{2} \sin \left(\frac{2 k \pi+\frac{2 \pi}{3}}{2}\right) & u_{1} \sin \left(\frac{2 k \pi+\frac{2 \pi}{3}}{2}\right) & \cos \left(\frac{2 k \pi+\frac{2 \pi}{3}}{2}\right)
\end{array}\right] .
$$




\section{Jafari and Molaei}

The first root for $k=0$ is

$$
A_{\circ}^{\frac{1}{2}}=\left[\begin{array}{cccc}
\frac{1}{2} & -\frac{\sqrt{3}}{2} & 0 & 0 \\
\frac{\sqrt{3}}{2} & \frac{1}{2} & 0 & 0 \\
1 & 1 & \frac{1}{2} & -\frac{\sqrt{3}}{2} \\
1 & -1 & \frac{\sqrt{3}}{2} & \frac{1}{2}
\end{array}\right],
$$

and the second one for $k=1$ is

$$
A_{1}^{\frac{1}{2}}=\left[\begin{array}{cccc}
-\frac{1}{2} & -\frac{\sqrt{3}}{2} & 0 & 0 \\
\frac{\sqrt{3}}{2} & -\frac{1}{2} & 0 & 0 \\
-1 & -1 & -\frac{1}{2} & \frac{\sqrt{3}}{2} \\
-1 & 1 & -\frac{\sqrt{3}}{2} & -\frac{1}{2}
\end{array}\right],
$$

Also, it is easy to see that $A_{\circ}^{\frac{1}{2}}+A_{1}^{\frac{1}{2}}=0$.

\section{Relations Between poWers of matrices}

Some relations between the powers of matrices associated with a semi-quaternion is sketched in the following theorem:

Theorem 8.1. Let $q$ be a unit semi-quaternion with the polar form $q=\cos \varphi+$ $\vec{w} \sin \varphi$, the matrix $A$ correspondnto $q$ and let $m=\frac{2 \pi}{\varphi} \in \mathbb{Z}^{+}-\{1\}$. Then $n \equiv p(\bmod$ $m)$ is true if and only if $A^{n}=A^{p}$.

Proof. The proof follows easily from the induction on $n$.

Example 8.1. Let $q=\frac{\sqrt{3}}{2}+\left(\frac{1}{2},-1,2\right)$ be a unit semi-quaternion. From the Theorem $8.1, m=\frac{2 \pi}{\pi / 6}=12$, so we have

$$
\begin{aligned}
A= & A^{13}=A^{25}=\ldots \\
A^{2}= & A^{14}=A^{26}=\ldots \\
& \ldots \\
A^{11}= & A^{23}=A^{35}=\ldots \\
A^{12}= & A^{24}=A^{36}=\ldots=I_{4} .
\end{aligned}
$$

Remark 8.1. The semi-quaternions are special cases of generalized quaternions if $\alpha=1, \beta=0$. (see $[10,11])$.

\section{Conclusion}

In this paper, we gave some of algebraic properties of the semi-quaternions and investigated the Euler's and De Moivre's formulae for these quaternions and also for the matrices associated with semi-quaternions. We also obtained $n$-th root of these matrices (Theorem 8.1). 


\section{Some Properties of Matrix Algebra of Semi-quaternions}

\section{REFERENCES}

[1] AdLer S. L., Quaternionic quantum mechanics and quantum fields, Oxford University Press inc., New York, 1995.

[2] Agrawal O. P., Hamilton operators and dual-number-quaternions in spatial kinematics, Mech. Mach. Theory. 22,no.6 (1987)569-575

[3] Сно E., De-Moivre Formula for Quaternions, Applied Mathematics Letters, 11(6) (1998)3335

[4] Dyachkova M., On Hopf bundle analogue for semiquaternion algebra, $10^{\text {th }}$ International Conference DGA, Olomouc, Czech Republic, 2007.

[5] Farebrother R.W., GroB J., Troschke S., Matrix Representaion of Quaternions, Linear Algebra and its Application, 362(2003)251-255

[6] Hamilton W.R., Lecture on Quaternions, Dublin : Hodges and Smith, 1853.

[7] Jafari M., Mortazaasl H., Yayli Y., De Moivre's Formula for Matrices of Quaternions, JP Journal of Algebra, Number Theory and appllication, Vol. 21, no.1 (2011) 57-67.

[8] Jafari M., Meral M., Yayli Y., Matrix Representaion of Dual Quaternions, Gazi University of Science, 26(4) (2013) 535-542.

[9] Kabadayi H., Yayli Y., De Moivre's Formula for Dual Quaternions, Kuwait Journal of Sci. \& Tech., Vol. 38, no.1 (2011)15-23

[10] Mamagani B.A, Jafari M., Some Notes on Matrix of Generalized Quaternions, International Research Journal of Applied and Basic Science, Vol. 7(14) (2013) 1086-1093.

[11] Mamagani B.A, Jafari M., On properties of Generalized Quaternion Algebra, Journal of Novel Applied Science, Vol. 12/2: 683-689.

[12] Mortazaasl H.,Jafari M., Yayli Y., Some Algebraic Properties of Dual Generalized Quaternions, Far East Journal of Mathematical Science, Vol. 69(2) (2012) 307-318.

[13] Mortazansl H., Jafari M., A Study on Semi-Quaternions Algebra in Semi-Euclidean 4Space, Mathematical Science and Application E-Notes, Vol. 1 (2) (2013) 20-27.

[14] Ozdemir M., The Roots of a Split Quaternion, Applied Mathematic Letters, 22(2009) 258263

[15] Ward J. P., Quaternions and Cayley Numbers Algebra and Applications, Kluwer Academic Publishers, London, 1997

[16] Yayli Y., Homothetic Motions at $E^{4}$. Mech. Mach. Theory, Vol. 27, no. 3 (1992)303-305

[17] Zhang F., Quaternions and Matrices of Quaternions, Linear Algebra and its Applications, 251(1997) 21-57 